\title{
Mobility of twin and phase boundaries
}

\author{
K. Bhattacharya, P. Purohit and B. Craciun ${ }^{1}$ \\ Division of Engineering and Applied Science, Mail Stop 104:44, \\ California Institute of Technology, Pasadena, CA 91125, U.S.A. \\ 1 Synopsys, 700 E. Middlefield Road, Mountain View, CA 94043-4044, U.S.A.
}

\begin{abstract}
This paper reviews some recent advances in understanding the mobility of twin and phase boundaries in martensites, and discusses the design of systematic experiments.
\end{abstract}

\section{INTRODUCTION}

Over the last decade, there has been much advance in the understanding of equilibrium martensitic microstructure, its relation to basic crystallography and its consequences on properties (see [1] for example). Much, however, remains unknown concerning the nucleation and evolution of microstructure, and the resultant hysteresis. These have been modelled at various levels of detail, virtually all models treat crucial aspects phenomenologically. Similarly, experimental studies are largely limited to macroscopic (stress-strain) hysteresis loops and few have considered the details of individual twin and phase boundaries. A predictive understanding of the factors that determine mobility and hysteresis is lacking.

Consider a rod of a martensitic material at rest above the transformation temperature. Suppose one end is held fixed, while the other is suddenly pulled with a given velocity. If this velocity is small, the rod behaves elastically with some shock (stress) waves propagating into it. But if the velocity is sufficiently high, the rod undergoes a phase transformation as martensitic domains nucleate and propagate. At what critical velocity do they nucleate? Where do they nucleate? How many nuclei? And how fast do they propagate? How is the behavior of single crystals different from that of polycrystals, $\mathrm{NiTi}$ from CuAlNi? Unfortunately, we know very little about these questions. This paper discusses some of the difficulties, and some recent attempts to overcome them. We concentrate on perhaps the easiest issue, the propagation velocity of a twin or phase boundary ${ }^{1}$ in an isothermal setting.

Let us consider this in the framework of Abeyaratne and Knowles [2]. To determine the propagation velocity of the interface, we first define a thermodynamic driving force $f$ acting on $i^{2}$. In the quasistatic setting, it is the rate of change of total energy with respect to the change in the position of the interface. In the dynamic setting, it is the rate of energy dissipated at the phase boundary divided by its velocity. This driving force can be calculated by knowing the states very close to the phase boundary. For example, in one dimension,

$$
f=[[W]]-\langle\sigma\rangle[[\gamma]]
$$

where $[[W]]$ denotes the jump in the stored energy across the phase boundary, $\langle\sigma\rangle$ denotes the average stress, and $[[\gamma]]$ denotes the jump in strain.

\footnotetext{
${ }^{1}$ Henceforth, we refer to both as phase boundary.

2This idea goes back to Eshelby [3] and is now understood in quite some sophistication $[4,5]$.
} 

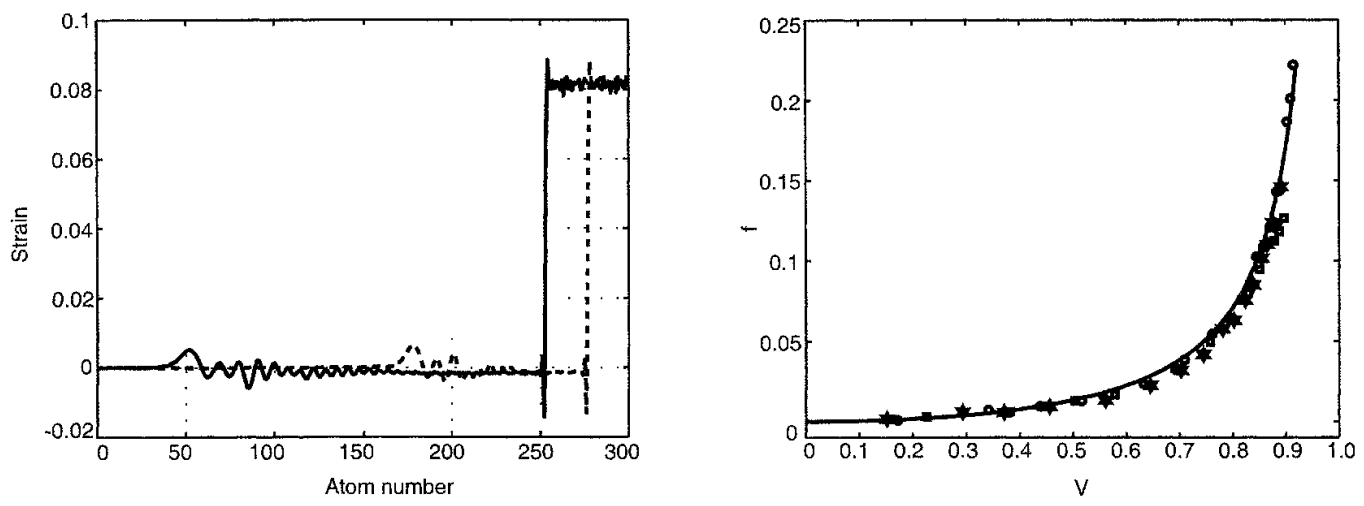

Figure 1. Left: Two snapshots of the chain when suddenly pulled from the right. Right: The kinetic relation that emerges from the chain. All in non-dimensional units.

We then postulate that the velocity of the phase boundary is a given function of this driving force:

$$
v=K(f)
$$

This relation $K$ between the driving force and the velocity is known as the kinetic relation and is regarded as a basic material property like the elastic modulus, transformation strain etc. Our interest is to find what determines the kinetic relation, and how we may measure it.

\section{DERIVING KINETIC RELATIONS}

A propagating twin or phase boundary involves a host of important physics at the microscopic scale. At the same time, not all of this microscopic physics is relevant. One can regard the kinetic relation as the distillation of the microscopic physics that are relevant to the macroscopic scale.

One approach at introducing this microscopic physics is to augment the usual equations of continuum physics by introducing viscosity and capillarity (strain-gradient correction), and to regard the phase boundary to be travelling waves or solitons of this equation $[6,7,8,9]$. Abeyaratne and Knowles [9] have shown that the velocity of a travelling wave depends on the end-states only through a very specific combination which is exactly the driving force. In short, the travelling waves define a kinetic relation.

A second approach is to study a discrete problem [10,11,12]. Purohit and Bhattacharya [13] recently considered a chain of masses connected through nonlinear bj-stable springs so that there are two equilibrium interatomic spacings. They found through simulation for a variety of initial and boundary conditions, that solutions quickly developed a travelling wave structure consisting of very sharp phase boundaries. Figure 1 (left) shows two snapshots of a simulation where the chain was initially in the low-strain phase and then subjected to a sudden pull on the right: note the clear sonic wave and sharp phase boundary. Furthermore, one can calculate the driving force across this phase boundary and plot it as a function of the phase boundary velocity. The results of numerous simulations - with different potentials, different initial and boundary conditions, different but small temperatures - are shown in Figure 1 (right): they all collapse on one curve which has the form

$$
f=c_{1}+\frac{c_{2}}{1-v^{2}}
$$

where $f$ is the (non-dimensionalized) driving force and $v$ is the velocity of the phase boundary non-dimensionalized by the speed of sound. In short, these simulations again define a kinetic relation. 

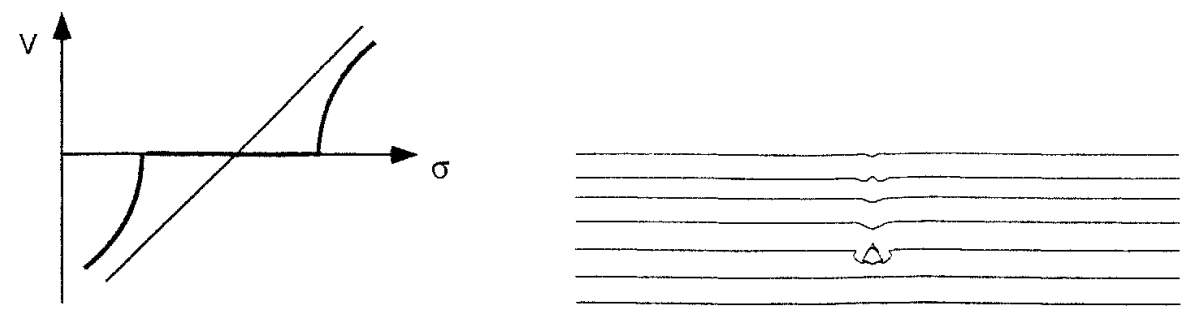

Figure 2. Left: The microscopic (thin line) and macroscopic (bold curve) behavior of a phase boundary propagating in a medium with defects. Right: Phase boundary propagating past a non-transforming precipitate.

The kinetic relations that one obtains from either of the approaches above - viscosity-capillarity and discrete - pass smoothly through the origin. Therefore, the hysteresis is completely ratedependent and vanishes as the loading rate decreases to zero. Experiments in martensites strongly suggest the converse: that the hysteresis has a rate-independent component. Therefore, phase boundaries must have a 'stick slip' character where we need to apply a critical driving force before they move. The lack of a rate-independent component appears to be an inherent feature of the viscosity-capillarity models, but appears to be an artifact of one-dimension in the discrete models. Specifically, in one dimension, one spring can go over the barrier at a time without requiring a change in any other interatomic spacing. This effectively reduces the barrier to zero. This is not be the case in higher dimensions.

Defects in the material can also contribute to the rate-independent component of hysteresis. Bhattacharya [14] studied the (quasi-static) propagation of a phase boundary in a heterogeneous one-dimensional media subjected to a given load $\sigma$. The microscopic propagation law had no rateindependent component as shown by the light line in Figure 2 (left); yet averaging the motion over a region gave rise to a significant 'stick-slip' character with a critical force below which there was no propagation followed by a very fast rise in velocity beyond this critical force as shown by the bold curve in Figure 2 (left). Roughly, the phase boundary had to go over the highest microscopic hurdle before it could have any macroscopic propagation, and would go quite rapidly as soon as it broke through the last hurdle.

The effect of defects can, however, be greatly exaggerated in one dimension as the phase boundary has no room to loop around it. Figure 2 (left) shows the results of a calculation where a phase boundary meets a (non-transforming) inclusion, but is able to propagate past it by skirting around it. The obstacle slows it down but does not pin it. Craciun and Bhattacharya [15] have used this model to show why $\mathrm{Ni}_{3} \mathrm{Ti}_{4}$ precipitates, which are introduced to precipitate harden $\mathrm{NiTi}$ by pinning dislocations, have only a minimal effect on the motion of twin boundaries.

\section{MEASURING KINETIC RELATIONS}

It is difficult to isolate a single phase or twin boundary in a situation where the driving force can be inferred even in carefully designed experiments [16]. Further, boundary effects are quite significant in multi-dimensional specimens [17]. Dynamic impact experiments avoid these difficulties [18], but are difficult to perform. Experiments in one-dimensional wires and bars under extension are a possibility, but it is difficult to observe the boundary.

Purohit and Bhattacharya $[19,20]$ have proposed some novel experiments using flexural deformations of wires and beams. Figure 3 (left) shows the behavior of a wire which is initially at rest in a stretched state and suddenly subjected to a downward velocity at one end. We have a shock (stress) wave, a kink or tangent discontinuity and a phase boundary. It turns out [19] that one can 

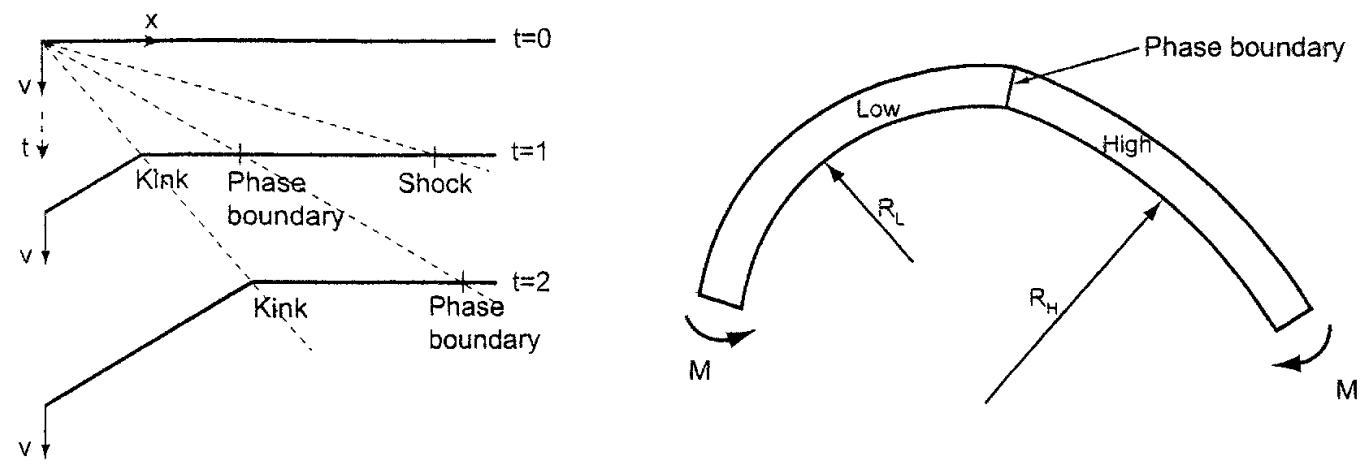

Figure 3. Left: A string suddenly pulled downward on the left end. Right: A beam made of a single crystal can have kinks and curvature discontinuities.

infer both the driving force and velocity of the phase boundary simply by measuring the velocity of the clearly visible kink. Thus this provides a novel experiment to measure the kinetic relation.

Similarly, beams made of martensites behave quite differently from classical beams. They can have significant stretch and shear; and discontinuities in them. For example, a beam made of a suitably oriented single crystal and subjected to a pure moment can deform with a kink and a discontinuous curvature [20] as shown in Figure 3 (right). Similarly cantilevers deform with a pronounced kink which can again be exploited to measure the kinetic relation [20].

A part from designing these experiments, the study of slender structures is important as most applications of shape-memory alloys are constructed from wires, strips and tubes and rely on flexural and torsional modes of deformation.

Acknowledgment We gratefully acknowledge the partial financial support of the Air Force Office of Scientific Research through a MURI grant (F 49620-98-1-0433).

\section{References}

[1] K. Bhattacharya, A theory of martensitic microstructure and its implications for the shape-memory effect, Oxford University Press, 2002, to appear.

[2] R. Abeyaratne and J.K. Knowles, Arch. Rat. Mech. Anal 114 (1991) 119-154.

[3] J.D. Eshelby, Solid State Physics 3 (1956) 17-144.

[4] R. Abeyaratne and J.K. Knowles, J. Mech. Phys. Solids 38 (1990) 345-360.

[5] M.E. Gurtin, Configurational forces as basic concepts of continuum physics, Springer Verlag (2000).

[6] M. Slemrod, Arch. Rat. Mech. Anal 83 (1983) 333-361.

[7] G.R. Barsch and J.A. Krumhansl, Phys. Rev. Lett. 53 (1984) 1069-1072.

[8] L. Truskinovsky Soviet Phys. Dokl. 27 (1985) 945-948.

[9] R. Abeyaratne and J.K. Knowles, STAM J. Appl. Math. 51 (1991) 1205-1221.

[10] J.A. Krumhansl and J.L. Schrieffer, Phys. Rev. B bf 11 (1975) 3535-3545.

[11] A.M. Balk, A.V. Cherkaev and I.L. Slepyan, J. Mech. Phys. Solids 49 (2001) 131-148 and 149-171.

[12] G. Puglisi and L. Truskinovsky, J. Mech. Phys. Solids 47 (2000) 1-27.

[13] P. Purohit and K. Bhattacharya, in preparation, 2002

[14] K. Bhattacharya, Proc. Royal Soc. London A 455 (1999) 757-766.

[15] B. Craciun and K. Bhattacharya, in preparation (2002).

[16] R. Abeyaratne, C. Chu and R.D. James, Phil. Mag. A 73(1996) 457-497.

[17] N.K. Simha and K. Bhattacharya, J. Mech. Phys. Solids 46 (1998) 2323-2359.

[18] J.C. Escobar and R.J. Clifton, J. Mat. Sci. Engng A 170 (1993) 125-142.

[19] P. Purohit and K. Bhattacharya, J. Mech. Phys. Solids, submitted (2001).

[20] P. Purohit and K. Bhattacharya, Int. J. Solids Structures, to appear (2002). 